\title{
High Sodium Diet and Autoimmune Diseases
}

\author{
Soonjae Hwang ${ }^{1}$, Hohyun Park ${ }^{2}$ and Ki-Jong Rhee ${ }^{1, \dagger}$ \\ ${ }^{I}$ Department of Biomedical Laboratory Science, College of Health Sciences, Yonsei University at Wonju, \\ Wonju, Gangwon-do 26493, Korea \\ ${ }^{2}$ Department of Biomedical Laboratory Science, Mokpo Science University, Mokpo-si, \\ Jeollanam-do 58644, Korea
}

\begin{abstract}
High sodium diet has been touted to be a major risk factor of disease in developed countries. The disease most closely associated with a high sodium diet is cardiovascular diseases. Autoimmune diseases are another broad spectrum of diseases that is associated with developed countries. In the past few years, several key scientific findings have revealed that a high sodium diet could also impact the pathogenicity of autoimmune diseases. In this review, we will highlight key results from such investigations and put it in context of high sodium diet and autoimmunity.
\end{abstract}

Key Words: Sodium chloride, Autoimmune disease, Th17 lymphocyte, SGK1

The prevalence of autoimmune diseases has steadily increased in developed countries. However, the various factors that predispose an individual to develop autoimmune diseases are unclear. Autoimmunity is characterized by a host's immune reaction to self-antigens resulting in uncontrolled immune process and subsequent damage to normal tissue structure and function (Davidson and Diamond, 2001). The autoimmune disease can manifest as rheumatoid arthritis, autoimmune thyroiditis, multiple sclerosis, systemic lupus erythematosus or type 1 diabetes. Many risk factors have been attributed to development of autoimmune disease including diet, gender, age, exposure to environmental agents and microbial infections (Vojdani, 2014). Among these various risk factors, one factor that has recently received close scrutiny is dietary sodium intake. One aspect of developed countries is the increased consumption of the

*Received: September 24, 2015 / Accepted: September 29, 2015

${ }^{\dagger}$ Corresponding author: Ki-Jong Rhee. Department of Biomedical Laboratory Science, College of Health Sciences, Yonsei University at Wonju, Wonju, Gangwon-do 26493, Korea.

Tel: +82-33-760-2445, Fax: +82-33-760-2561

e-mail: kjrhee@yonsei.ac.kr

(C) The Korean Society for Biomedical Laboratory Sciences. All rights reserved.
"Western" diet often equated with increased consumption of processed and "fast foods". Considering that many fast foods have high levels of dietary sodium, the effects of sodium consumption on health has garnered particular interest by scientific and clinical researchers (Powles et al., 2013). According to one report, the sodium content in fast foods was 100-fold higher than comparable foods prepared at home (Brown et al., 2009). Further, this study showed that the average consumption of dietary sodium was twofold higher in Asian countries compared to Western countries. Several studies have highlighted the correlation between high-sodium diet and incidence of autoimmune disease. In this short review, we will describe scientific research that suggests one possible mechanistic explanation on how dietary sodium may promote autoimmune diseases.

Most researchers agree that the adaptive immune system, especially $\mathrm{T}$ helper (Th) lymphocytes, plays a pivotal role in pathogenesis of many autoimmune diseases. There are three types of Th lymphocytes which have been identified on the basis of the specific cytokines they produce and depending on the invading pathogen, the way in which these cytokines modulate subsequent immune reactions. Of these 


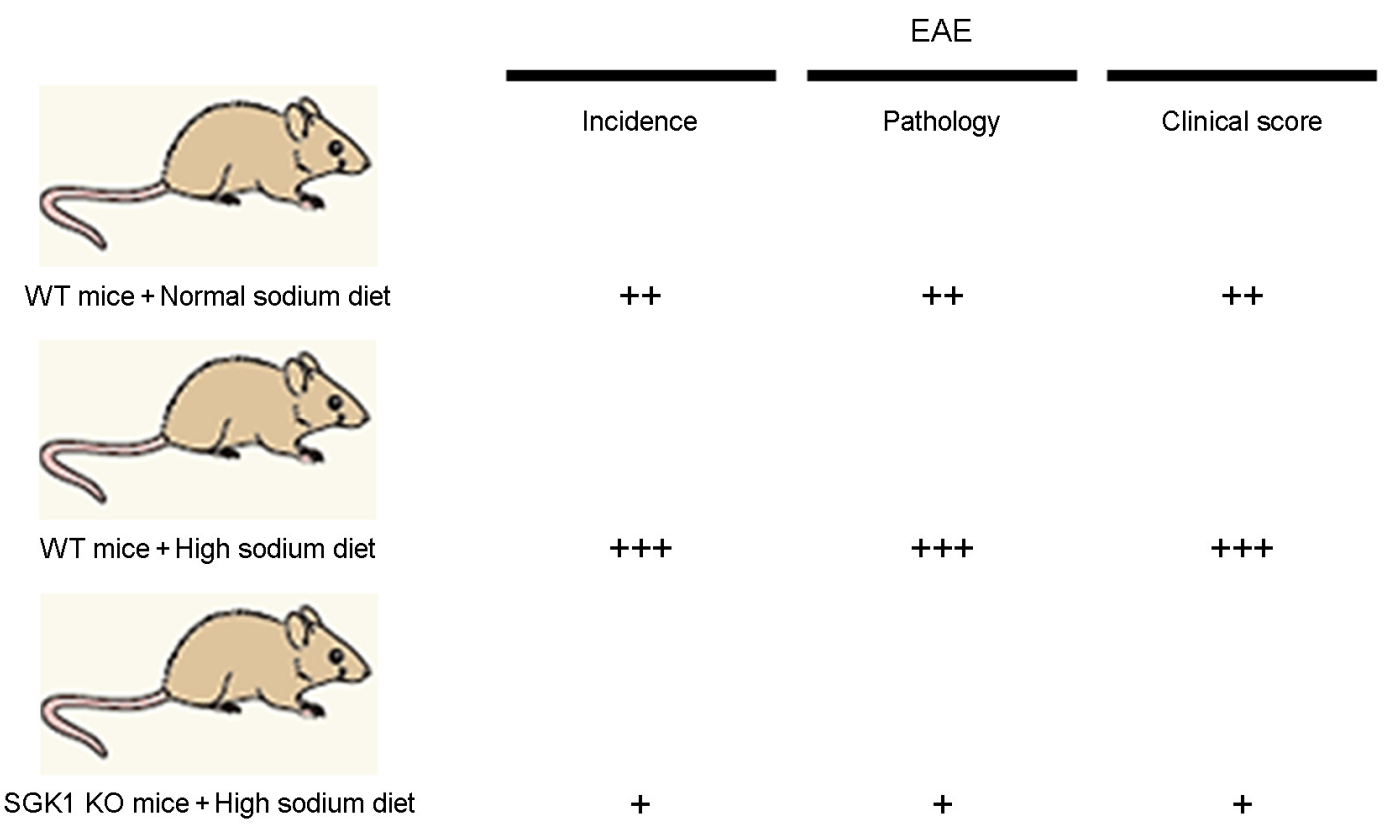

Fig. 1. High sodium diet aggravates EAE pathogenesis in a murine model of autoimmunity. WT or SGK1 KO mice were given a normal sodium diet or high sodium diet and EAE incidence, pathology and clinical score examined.

three type of Th lymphocytes, Th17 lymphocytes secrete interleukin-17 (IL-17) as well as other cytokines which function to promote an aggressive immune response against bacteria and fungi infections. These cytokines induce recruitment of other immune cells to infected tissues to coordinate the host's defense. Generally, the Th17 lymphocytes undergo apoptotic cell death after eliminating the pathogen of interest. However, excessively activated and/or persistent Th17 lymphocytes can exacerbate tissue damage and disrupt homeostasis, thereby conferring susceptibility to autoimmune disease. During the past decade, results from numerous scientific data have strongly suggested that Th17 polarization is intimately involved in promotion of many autoimmune diseases.

In 2013, two independent research groups have reported that sodium aggravates autoimmune disease by promoting Th17 polarization (Kleinewietfeld et al., 2013; Wu et al., 2013). Although the two research groups differ slightly in the methodology, both groups utilized the experimental autoimmune encephalomyelitis (EAE) mouse model for multiple sclerosis. In this model, mice are immunized with myelin oligodendrocyte glycoprotein (MOG) which results in generation antigen-specific pathogenic Th17 lymphocytes and subsequent elaboration of encephalomyelitis. This model is mostly applied in mice and is generally considered to be a prototype for $\mathrm{T}$ lymphocyte mediated autoimmune disease. In essence, both groups reached the conclusion that a highsodium diet aggravates neuropathology in the EAE model. Wild-type (WT) mice injected with MOG developed the canonical symptoms of EAE when given a normal sodium diet (Fig. 1). However, when the MOG-injected WT mice were given a high sodium diet, they exhibited higher incidence of EAE, more severe pathology and higher clinical scores. These mice had higher levels of Th17 lymphocytes compared to WT mice given a normal sodium diet. Mechanistically, they showed that sodium activates the signaling protein p38 MAPK which in turn regulates SGK1 and NFAT5. Their results indicate that SGK1 is induced in Th17 lymphocytes and that lack of SGK1 in genetically deficient mice led to decreased neuropathology in the EAE model (Fig. 1). Consistent with this idea, MOG-injected SGK1 KO mice developed decreased incidence of EAE, decreased 
pathology and decreased clinical scores compared to WT mice given a high sodium diet. Wu et al. further showed that in vitro culture of undifferentiated Th lymphocytes in a high-sodium milieu enhanced Th17 polarization suggesting that the effect of sodium could act directly on Th lymphocytes (Wu et al., 2013). Thus the two research groups have come to the same conclusion that increased salt concentrations aggravate autoimmune disease by enhancing the stimulation of the production of interleukin-17-producing Th17 lymphocytes from naïve Th lymphocytes. Although the effects of a high-salt diet on initiation of EAE are evident in both in vitro and in vivo model systems, the medical implications with humans are still relatively unclear.

Many attempts have been made to evaluate the effect of high-sodium diet on the immune system. For example, in one longitudinal study, Yi et al. evaluated the clinical impact of a quantified high salt diet on healthy people (Yi et al., 2015). Patients were given a protocol of fixed salt intake of 6,9 , or 12 grams of salt per day for several months and thereafter examined the relationship between salt intake levels and changes in the immune system. They collected blood samples and monitored peripheral leukocyte phenotype changes. This group reported that the subjects on the high sodium diet of 12 grams per day displayed a significantly higher number of monocytes compared with the same subjects on a lower sodium diet. Moreover, the decreased sodium intake was accompanied by decreased production of pro-inflammatory cytokines such as interleukin-6 and interleukin-23, along with increased production of antiinflammatory cytokine interleukin-10. Of interest, both interleukin-6 and interleukin-23 are critical factors required for differentiation of Th17 lymphocytes from naïve Th cells (Lee et al., 2012). In another study, it was reported that a high sodium diet in rats leads to stimulation of macrophages (Machnik et al., 2009). Also, Rossol et al. found that coculture of blood monocytes from rheumatoid arthritis patients with naive Th cells were potent inducers of Th17 lymphocytes (Rossol et al., 2012). Taken together, these discoveries, albeit preliminary, suggest the tantalizing possibility that a high-sodium diet can modulate the immune system which then provides an environment conducive to skewing of a Th17 immune response further promoting the initiation and/ or aggravating Th17 lymphocyte-induced diseases. Until recently, the vast majority of studies on the effects of highsodium diet have been on sodium uptake and cardiovascular disease (Strazzullo et al., 2009). Although the implication of these studies on humans still remain a matter of debate, more research on the multifactorial aspects of a high sodium diet on the immune system is warranted.

\section{Conflict of interest}

The authors declare that they have no conflict of interest.

\section{REFERENCES}

Brown IJ, Tzoulaki I, Candeias V, Elliott P. Salt intakes around the world: implications for public health. Int J Epidemiol. 2009. 38: 791-813

Davidson A, Diamond B. Autoimmune diseases. N Engl J Med. 2001. 345: 340-350.

Kleinewietfeld M, Manzel A, Titze J, Kvakan H, Yosef N, Linker RA, Muller DN, Hafler DA. Sodium chloride drives autoimmune disease by the induction of pathogenic $T_{H} 17$ cells. Nature. 2013. 496: 518-522.

Lee Y, Awasthi A, Yosef N, Quintana FJ, Xiao S, Peters A, Wu C, Kleinewietfeld M, Kunder S, Hafler DA, Sobel RA, Regev A, Kuchroo VK. Induction and molecular signature of pathogenic $\mathrm{T}_{\mathrm{H}} 17$ cells. Nat Immunol. 2012. 13: 991-999.

Machnik A, Neuhofer W, Jantsch J, Dahlmann A, Tammela T, Machura K, Park JK, Beck FX, Muller DN, Derer W, Goss J, Ziomber A, Dietsch P, Wagner H, van Rooijen N, Kurtz A, Hilgers KF, Alitalo K, Eckardt KU, Luft FC, Kerjaschki D, Titze J. Macrophages regulate salt-dependent volume and blood pressure by a vascular endothelial growth factor-Cdependent buffering mechanism. Nat Med. 2009. 15: 545-552.

Powles J, Fahimi S, Micha R, Khatibzadeh S, Shi P, Ezzati M, Engell RE, Lim SS, Danaei G, Mozaffarian D. Global, regional and national sodium intakes in 1990 and 2010: a systematic analysis of $24 \mathrm{~h}$ urinary sodium excretion and dietary surveys worldwide. BMJ Open. 2013. 3: e003733.

Rossol M, Kraus S, Pierer M, Baerwald C, Wagner U. The CD14 ${ }^{\text {bright }}$ CD16+ monocyte subset is expanded in rheumatoid arthritis and promotes expansion of the Th17 cell population. Arthritis Rheum. 2012. 64: 671-677.

Strazzullo P, D'Elia L, Kandala NB, Cappuccio FP. Salt intake, stroke, and cardiovascular disease: meta-analysis of prospective 
studies. BMJ. 2009. 339: b4567.

Vojdani A. A potential link between environmental triggers and autoimmunity. Autoimmune Dis. 2014. 2014: 437231.

Wu C, Yosef N, Thalhamer T, Zhu C, Xiao S, Kishi Y, Regev A, Kuchroo VK. Induction of pathogenic $\mathrm{T}_{\mathrm{H}} 17$ cells by inducible salt-sensing kinase SGK1. Nature. 2013. 496: 513-517.
Yi B, Titze J, Rykova M, Feuerecker M, Vassilieva G, Nichiporuk I, Schelling G, Morukov B, Chouker A. Effects of dietary salt levels on monocytic cells and immune responses in healthy human subjects: a longitudinal study. Transl Res. 2015. 166: 103-110. 